pressures than those studied in the non-drug studies. The blood pressures before treatment of patients in the drug and non-drug studies were not significantly different (systolic blood pressure, $t=0.69$, df 98 ; diastolic blood pressure, $t=0.43$, df 109).

In conclusion, we suggest that rather than see the outcome of our analysis primarily in terms of non-pharmacological treatments being shown to be less effective than drug treatments, the most important outcome is that several non-drug treatment modalities can be seen to have produced substantial and lasting reduction in blood pressure. These benefits are perhaps overall not as great as those of the drug studies, but are significantly greater than those of the placebo studies and are of appreciable clinical importance. We would certainly agree that many mildly hypertensive patients might be treated effectively by non-drug techniques, and indeed have concluded that: "Weight loss, progressive muscle relaxation, and yoga could provide competitive alternatives to step 1 drug treatment," and should be considered by the doctor.

GAVIN ANDREWS STEPHEN MACMAHON ANNe Austin

School of Psychiatry,

University of New South Wales, Prince Henry Hospital,

Little Bay 2036

Australia

DONALD ByRne

Department of Psychology,

Australian National University,

Canberra,

Australia

\section{Sinus arrest during treatment with amiodarone}

SIR,-Beginning with the initial report of sinus arrest during treatment with amiodarone by $\mathrm{Dr} B$ McGovern and others (16 January, p 160), there has been a continuing discussion (27 February, p 664, and 10 April, p 1120) about the ability of amiodarone by itself to cause sinus arrest, since all previously reported patients have also been receiving digoxin. The question has been raised of whether the observed sinus arrest may be attributable to the digoxin or the amiodarone alone or to the combination of the two. We describe a patient who developed sinus arrest with only amiodarone.

A 56-year-old white woman developed recurrent, refractory ventricular tachycardia about six weeks after an inferior wall myocardial infarction. On transfer to our institution she had been cardioverted and/or defibrillated more than 100 times. Her baseline electrocardiogram showed sinus rhythm, normal conduction intervals, old anterior and old inferior wall myocardial infarctions, and non-specific lateral ST segment and $T$ wave abnormalities. Her QT interval without any antiarrhythmic drugs was 0.44 seconds. The ventricular tachycardia was polymorphous, with rates varying between 250 and 350 beats $/ \mathrm{min}$. In the setting of multiple cardiac arrests the patient developed moderate congestive heart failure which required a brief period of inotropic support with dobutamine and subsequently digitalis, but she was eventually stabilised on diuretics and nitrates. Because her ventricular arrhythmias worsened with procainamide and were refractory to lignocaine, bretylium, diphenylhydantoin, mexiletine, and atrial and ventricular pacing, amiodarone was begun two months after her acute myocardial infarction. She received $1 \mathrm{~g}$ a day for two weeks, and then was maintained on $600 \mathrm{mg} /$ day. Digoxin $0.125 \mathrm{mg}$ by mouth four times daily was stopped 10 days into the course of amiodarone because of sinus bradycardia of 40 beats/min. The digoxin level eight hours after the last dose was $180 \mu \mathrm{g} / \mathrm{l}$ and the creatinine was $70 \mu \mathrm{g} / \mathrm{l}$.

Twenty days after the amiodarone was started and 10 days after her last dose of digoxin the patient developed sinus bradycardia of 30 beats/ $\mathrm{min}$, and a 12-second period of sinus arrest was associated with loss of consciousness and a brief seizure. Temporary and subsequently permanent pacing were instituted, and she has remained stable since then on amiodarone with the pacemaker.

To our knowledge this represents the first case of sinus arrest in a patient taking only amiodarone. It supports the suggestion of Dr McGovern and others that this is a potential side effect of amiodarone treatment and furthermore is not surprising in light of its recognised ability to cause extreme sinus bradycardia ${ }^{1}$ and to impair sinus node function as measured by corrected sinus node recovery time. ${ }^{2}$ The response of this patient's very rapid polymorphous ventricular tachycardia to the amiodarone has been gratifying. The occurrence of sinus arrest after 20 days of treatment, however, is of obvious concern and suggests that a longer monitoring period may be necessary. At the very least, sinus arrest needs to be recognised as a potential complication of amiodarone treatment, especially in patients who may develop recurrent symptoms on amiodarone that would otherwise be attributable to a recurrence of their tachycardia.

WILliam N BRODINE JOHN DESANTIS

Albany Medical College of Union University, Albany New York 12208

' Heger J, Prystowsky E, Jackman W, Naccarelli G, Warfel K, Rinkenberger R. N Engl f Med 1981

305:539-45.
Toubal P, Atallah G, Gressard A, Kirkorian G. Br Heart 7 1979;42:573-8.

\section{Subdural haematoma as a complication of spinal anaesthetic}

SIR,-We were interested to read Drs Paul Newrick and David Read's report of subdural haematoma after dural puncture in two previously healthy individuals ( 31 July, p 341). We feel, however, compelled to write to highlight the far-reaching implications that were not made obvious in this reportnamely, the well-known adage that prevention is better than cure.

That the postspinal headache is due to leakage of cerebrospinal fluid is well documented $^{12}$ and that the incidence of postspinal headache correlates well with the size of the needle used, and hence the dimensions of the hole or rent in the dura, has also been shown $\left(77.5 \%\right.$ with an 18 -gauge Touhy needle). ${ }^{3}$ In this hospital a 16-gauge Touhy needle is routinely used for obstetric epidural analgesia and our overall incidence of inadvertent dura tap of $3 \%-4 \%$ compares favourably with other hospitals where there is an active teaching programme. The accidental occurrence of a severe headache in an otherwise normal obstetric patient who wishes to enjoy her new baby is disastrous. It seems entirely reasonable therefore, to perform simple measures to minimise the loss of cerebrospinal fluid. These measures include performing epidural puncture at an adjacent lumbar interspace to provide continuous epidural block and forceps delivery to minimise inadvisable straining, keeping the patient in bed for 24 hours in the supine position whenever possible, and ensuring an adequate fluid intake. ${ }^{4}$

Moir $^{4}$ and Crawford ${ }^{5}$ have shown significant advantages in injecting $40-60 \mathrm{ml}$ o sterile Ringer lactate or normal saline through the catheter after delivery, and allowing $1000 \mathrm{ml}$ of sterile Ringer lactate to drip slowly into the epidural space over the next 24 hours. If a postspinal headache still occurs after these measures an epidural blood patch is advocated. Indeed, Digiovani et $a l^{6}$ suggest that an epidural blood patch should be performed routinely at the time of lumbar puncture as a prophylaxis against postspinal headache. In the light of the possibility of a subdural haematoma we would suggest that these measures, far from being recommended to prevent postspinal headache should be mandatory to prevent permanent neurological sequelae or even death.

A more careful review of the 12 reported cases of this rare complication reveals that in only one case was strict bed rest for 24 hours ordered and the same case represented the only patient in whom a fluid regimen of 6 litres in 48 hours was instituted. Only one patient was treated with a epidural blood patch, 38 days after spinal tap, and chronic subdural haematomas were confirmed at necropsy two days later. In view of this it would be interesting to know the following omissions from the report. Firstly, was either patient treated at an early stage with the standard minimum regimen quoted for postpinal headache ? Secondly, what measures, if any, were taken in case 1 to prevent subarachnoid hypotension mediated by straining in the second stage of labour? Thirdly, were any other active interventions to treat postspinal headache performed ?

If all these methods for the reduction of postspinal headache were used in these cases then the authors can claim to have described the first published cases of subdural haematoma after correctly managed postspinal headache. We suspect, however, that this is not the case and must therefore modify their comment to read "subdural haematoma can undoubtedly occur in fit healthy patients after spinal puncture if early prophylactic measures against cerebrospinal fluid leakage are not instituted."

C P O GARRETT S N BOLSIN

Department of Anaesthetics, University College Hospital, University Coll 1 Brown BA, Jones OW. F Neurosurg 1962;19:349. ${ }^{3}$ Crawford JS. Br $\mathcal{Y}$ Anaesth 1972;44:66 Moir DD. Obstetric anaesthesia and analgesia. London Cassell, 1980:199.

Crawford JS. Br F Anaesth 1972;44:598.

giovani AJ, Galbert MW, Wahle WM. Anesth

*We sent a copy of this letter to the authors, who reply below.-ED, $B M \mathcal{H}$.

SIR,-We are grateful for the opportunity to reply to the criticisms of Dr Garrett and Dr Bolsin, and we would wholeheartedly agree with them that prevention is better than cure. We are, however, not so sanguine about the prospects for this in the context of subdural haematoma after lumbar puncture.

Much has been written about the management of postspinal headache, but the evidence does not confirm the efficacy of regimens often quoted of fluid intake, ${ }^{1}$ administration of 1 - 